\title{
Ki-67 Expression In Hepatocellular Carcinoma Developed On A Liver Girrhosis
}

${ }^{1}$ Clinical and Emergency County Hospital of Constanta, Constanța

${ }^{2}$ Department of Public Health, Faculty of Medicine, "Ovidius" University, Constanța, ${ }^{3}$ Department of Hygiene, Faculty of Medicine, "Ovidius" University , Constanța

\begin{abstract}
Hepatocellular carcinoma is one of the most common cancers worldwide and a major cause of death in many countries. To evaluate the clinicopathological correlations of proliferative activity in patients with hepatocellular carcinoma, Ki-67 antigen expression was examined using immunohistochemical staining with monoclonal antibody MIB1. Consecutive 40 patients who had undergone surgical resection for the treatment of HCC from January 2005 to December 2009 were enrolled in our study. The study group comprised 32 men and 8 women ranging from 27 to 76 (mean age, 54.6 $\mathrm{SD} \pm 11.7)$ years of age. Expression of $\mathrm{Ki}-67$ was negative in $22.5 \%$ of the 40 cases and $\mathrm{Ki}-67$ positive expression "+", "++", "+++" was found in 11, 7 and 13 cases of hepatocellular carcinoma respectively. There are no statistically significant differences of the Ki-67 mean between age, sex distribution, dimension of the tumors, pattern of the tumor and Edmondson -Steiner grading.
\end{abstract}

$$
\text { Key words: hepatocellular carcinoma, }
$$
imunohistochemical expression, Ki-67

Elena Mocanu MD

Faculty of Medicine, "Ovidius” University, Constanta Aleea Universității nr.1, 900437 Constanța, România Tel: +40-722-664-768

E-mail: elenamocanu80@yahoo.com

\section{Introduction}

Hepatocellular carcinoma (HCC) is one of the most common cancers worldwide and a major cause of death in many countries [1].

Cell proliferation is considered to play an important role in the several steps of carcinogenesis process. Ki-67 is one of the many antigens protein has been used as proliferation marker for cancer cells [2]. Ki-67 is located in cell nuclei $[3,4]$.

\section{Material and Methods}

To evaluate the clinicopathologic correlation of proliferative activity in patients with hepatocellular carcinoma, Ki-67 antigen expression was examined using immunohistochemical staining with monoclonal antibody MIB1.

Consecutive 40 patients who had undergone surgical resection for the treatment of HCC at Fundeni Clinical Institute-Department of General Surgery and Liver Transplantation January 2005 to December 2009 were enrolled in our study. The macro- and microscopic examination and immunohistochemical analysis of the pieces of hepatic resection were performed at the Pathological Anatomy department of the same institution

Clinicopathological parameters were analyzed included sex, age, liver pathology (hepatitis, cirrhosis), tumor size, tumor differentiation (Edmondson-Steiner grading) and tumor pattern. 
Table I - Characteristics of Ki-67 in this study

\begin{tabular}{|l|l|l|l|}
\hline $\begin{array}{c}\text { Name of } \\
\text { antibody }\end{array}$ & Specificity & Dilution & $\begin{array}{c}\text { Secondary } \\
\text { antibody }\end{array}$ \\
\hline Ki-67 & $\begin{array}{l}\text { Monoclonal } \\
\text { Mouse anti } \\
\text { human }\end{array}$ & $1: 50$ & $\begin{array}{l}\text { Goat anti } \\
\text { mouse }\end{array}$ \\
\hline
\end{tabular}

Surgical liver resections for HCC from 40 patients ( 32 men, 8 women, mean age, $54.6 \mathrm{SD} \pm 11.7$, range $27-76$ years) were retrospectively studied.

HCC was associated with cirrhosis (19 cases) or chronic hepatitis due to HCV (8 cases) or HBV infection ( 27 cases), to alcohol alone (5 cases).

For Ki-67 immunostaining, deparaffinized sections were pretreated in $0.01 \mathrm{~mol} / \mathrm{L}$ citrate buffer, $\mathrm{PH}=2.5$. Immunolabeling was performed using the immunohistochemistry automat, who used the avidinbiotin peroxidase complex (ABC) method with 3,3-diaminobenzidine as chromogen and hematoxylin for counterstaining.

Immunohistochemistry staining was performed with SP three-step method using the monoclonal antibodies listed in table 1.

Were identified and used for simplicity four degrees for Ki 67: “"-" < 24\%, "+" $=25 \%-50 \%$, "++" $=51 \%-74 \%, "+++">75 \%$.

\section{Statistical analysis}

To perform the statistical analyses we used the software package SPSS 19.0. Results of quantitative data in immunohistochemical analyses were expressed as mean \pm one SE. Correlation Pearson test and $\chi^{2}$ test were used to examine the association between clinico-pathological features and the biomarkers expression. The significant level was defined as a pv $<0.05$.

\section{Results}

It can be noticed that the imunohistochemical expression of Ki-67 was negative in $22.5 \%$ of the 40 cases and Ki-67 positive expression "+", "++", "“++" was found in 11, 7 and 13 cases of hepatocellular carcinoma respectively (Figure 1).
For the female (Figure 2), imunoexpression predominant were intense positive in $50 \%$ of cases, followed by "+" in $25 \%$ of cases and $12,5 \%$ for " ++ " and negative. Regarding the distribution in males, we find 9 cases in which the expression Ki67 was "+", 9 cases with "++", 6 cases with "+++" and 8 cases without imunoexpression. Hi square calculated (1.558) is less than hi spreadsheet square (7.82) for three degrees of freedom and statistical significance level of $5 \%$, from which we conclude that there was no statistically significant correlation( $\mathrm{pv}=0.67)$ between gender distribution and ki-67 immunoexpression.

To analyse the association level between the Ki-67 values and the age of the people diagnosed with

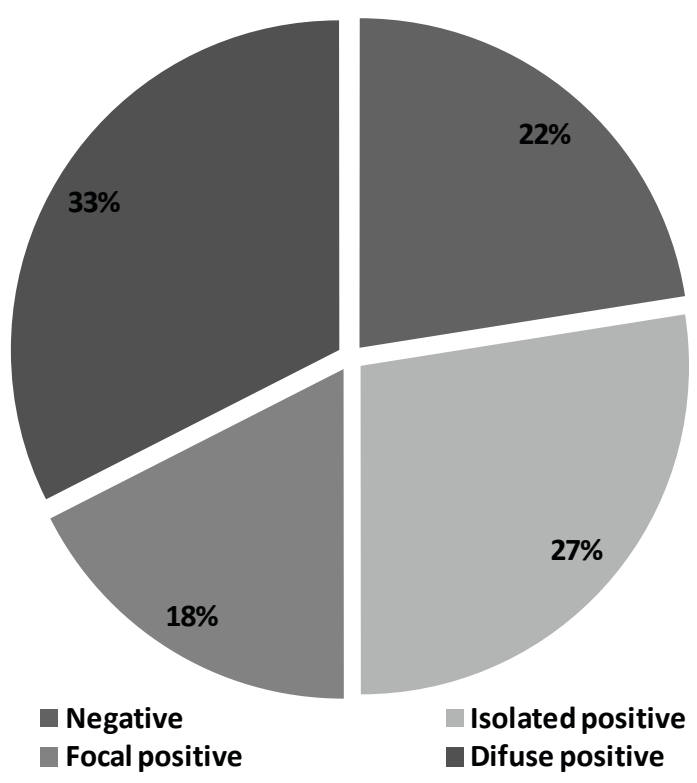

Figure 1 - Imunohistochemical expression of Ki-67

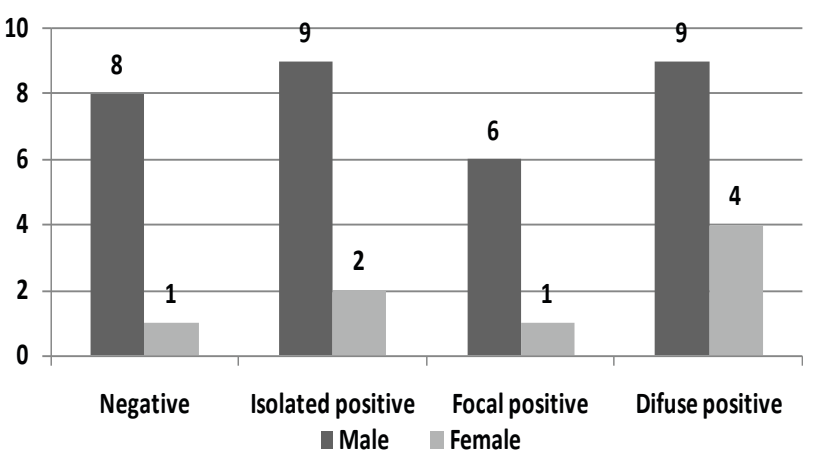

Figure 2 - Imunoexpression of Ki-67 related with sex distribution 


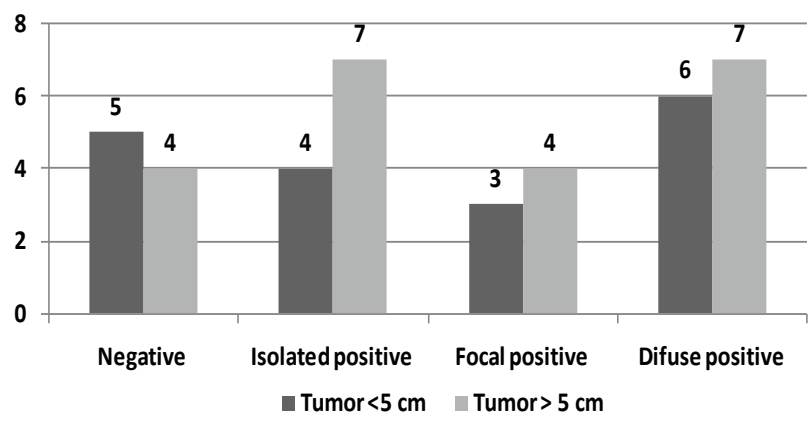

Figure 3 - Imunoexpression of Ki-67 related with tumor size distribution

HCC, the Pearson correlation factor was used. The results represent a positive correlation, insignificant for the two characteristics $(\mathrm{r}=0.109, \mathrm{pv}=0.504$, bilateral).

According to tumor size, the 40 cases were divided into two groups, i.e., $<5 \mathrm{~cm}(\mathrm{n}=18)$ and $\geq 5$ $\mathrm{cm}(\mathrm{n}=22)$ (Figure 3$)$.

Among the 18 HCCs measuring less $5 \mathrm{~cm}$, 6 case $(33,3 \%)$ were "+++", 3 cases $(16,6 \%)$ were "++" , 4 case $(22,2 \%)$ were "+" and 5 cases were "_.".

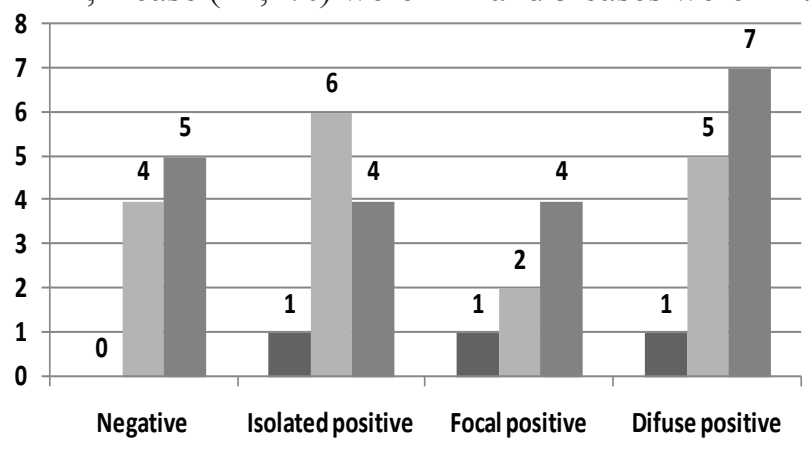

-Well differntiated Moderately differentiated $\square$ Poorly differentiated

Figure 4 - Edmondson Steiner grading

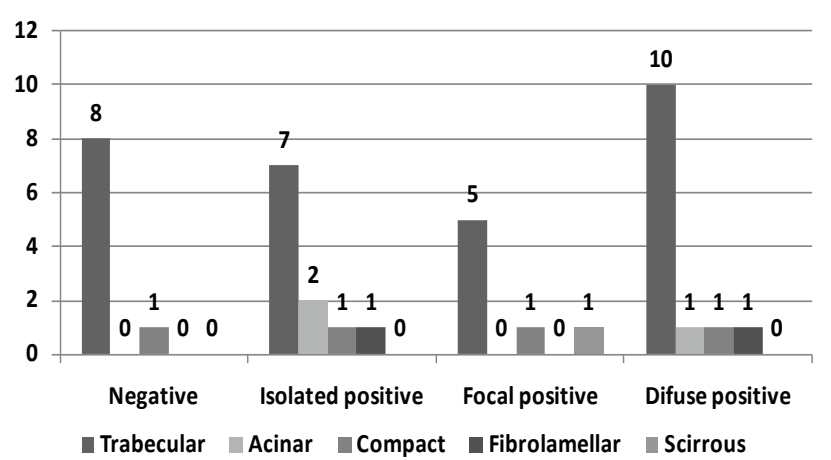

Figure 5 - Expression of Ki-67 in different hepatocellular carcinoma pattern

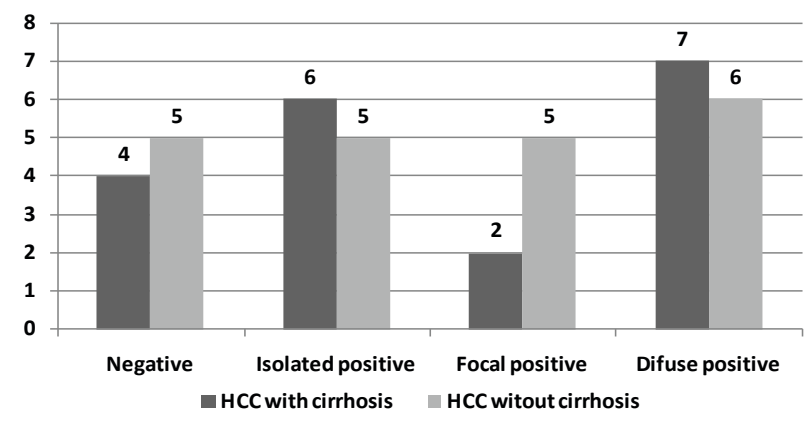

Figure 6 - Expression of Ki-67 in the HCC with cirrhosis and HCC without cirrhosis groups

Among the $22 \mathrm{HCCs}$ larger than $5 \mathrm{~cm}$ in diameter, 7 cases were "+++", 7 cases were "+", 4 cases were "++" and 4 cases were "-."

By applying the Pearson correlation test no statistically significant association between the Ki-67 values and dimension of tumors $(\mathrm{r}=0.035, \mathrm{pv}=0.832)$ can be made.

Among the 17 moderately differentiated HCCs, 5 cases $(29.4 \%)$ were intense positive with "+++", 2 cases (11.7\%) were positive "++", 6 cases $(35.2 \%)$ were positive "+", and 4 cases $(23.5 \%)$ were negative.

Among the 20 poorly differentiated HCCs 7 cases $(35 \%)$ were intense positive with"+++", 4 cases $(20 \%)$ were positive "++", 4 cases $(20 \%)$ were positive "+", and 5 cases (25\%) were negative.

In regard to the relationship between $\mathrm{Ki}$ 67 expression and histological grade (Figure 4) of HCCs in the 40 nodules, $\chi^{2}$ calculated (2.47) is less than $\chi^{2}$ spreadsheet $(12.59)$ for $\mathrm{df}=6$ and statistical significance level of $5 \%$, from which we concluded that there was no statistically significant correlation( $\mathrm{pv}=0.872$ )

Trabecular pattern represents $75 \%$ of the cases (Figure 5). Hi square calculated (9.6) is less than hi spreadsheet square (21.03) for 12 degrees of freedom and statistical significance level of 5\%, from which we conclude that there was no statistically significant correlation( $\mathrm{pv}=0.651)$ between pattern of hepatocarcinomas and ki-67 immunoexpression.

In figure no. 6 observed that in the HCC with cirrhosis immunoreactivity for 4 cases were negative, 6 cases were isolated positive, 2 cases focal positive and 7 cases were difuse positive.

$\chi^{2}$ calculated (1.46) is less than $\chi^{2}$ spreadsheet 
(7.82) for three degrees of freedom and statistical significance level of 5\%, from which we concluded that there was no statistically significant correlation( $\mathrm{pv}=0.69$ ) between presence of liver cirrhosis and ki67 immunoexpression.

\section{Discussion}

The expression of the human $\mathrm{Ki}-67$ protein is strictly associated with cell proliferation. The fact that the $\mathrm{Ki}-67$ protein is present during all active phases of the cell cycle, but is absent from resting cells, makes it an excellent marker for determining the socalled growth fraction of a given cell population. Ki67 protein expression is an absolute requirement for progression through the cell-division cycle [4].

The present study drew special attention to the relationship between the histological grades of $\mathrm{HCC}$ and $\mathrm{Ki}-67$, and we examined $\mathrm{Ki}-67$ expression in various types of HCCs, including small to large sized, well to poor differentiated HCCs and HCC with cirrhosis and HCC without liver cirrhosis.

In a study of patients who had undergone resection for $\mathrm{HCC}$, higher levels of expression of Ki67 in tumor tissue were found to be associated with early disease recurrence [5] and a higher tumor grade [6]

No significant relationship between sex and Ki-67 labeling index was found $(p>0.05)$. In the literature, it is known that the prognosis is better and the ratio of reccurrence lower in women than men [7].

$47,5 \%$ of $40 \mathrm{HCC}$ cases in this study were suffering from cirrhosis. However the level of staining was no significant different between the HCC with cirrhosis and HCC without cirrhosis groups.

In our study Ki-67 expression was the highest in poorly differentiated HCC, and it was followed by moderately differentiated HCC, and then welldifferentiated HCC, but in study of Soyouer [8], Ki67 expression was the highest in poorly-differentiated HCC, and it was followed by well - differentiated $\operatorname{HCC}(\mathrm{t}=2.96 ; \mathrm{p}<0.01)$.

\section{Conclusions}

By applying correlation Pearson test and hi square test for examine the association between clinico-pathological features and the Ki-67 immunoexpression we concluded:

1. There are no statistically significant differences of the Ki-67 mean between males and females.

2. Age is not correlated to the value of Ki-67.

3. Ki-67's value is not correlated to the dimension of the tumors in a statistically significant manner.

4. There are no statistically significant differences between the mean Ki-67 in relation to the histological grading and pattern.

\section{References}

1. FERENCIP,FRIEDM,LABRECQUED, BRUIX J, SHERMAN M.: World Gastroenterology Organisation Guidelines and Publications Committee. Hepatocellular carcinoma (HCC): a global perspective. J Gastrointestin Liver Dis. 2010;19(3):311-317.

2. LIN GY, CHEN ZL, LU CM, LI Y, PING XJ, HUANG R.: Immunohistochemical stud y on p53, H-rasp21, c-erbB-2 protein and PCNA expression in $\mathrm{HCC}$ tissues of Han and minority ethnic patients. World J Gastroenterol. 2000; 6(2): 234- 238 .

3. SCHMITT-GRAFF A, ERTELT V, ALLGAIER HP, KOELBLE K.: Cellular retinol-binding protein-1 in hepatocellular carcinoma correlates with beta-catenin, Ki-67 index, and patient survival. Hepatology 2003; 38: 470-480

4. JUN CUI, BAO-WEI DONG, PING LIANG, XIAO-LING YU, DE-JIANG YU: Effect of c-myc, Ki-67, MMP-2 and VEGF expression on prognosis of hepatocellular carcinoma 
patients undergoing tumor resection, World $\mathrm{J}$ Gastroenterol 2004;10(10):1533-1536

5. D'ERRICO A, GRIGIONI WF, FIORENTINO M, BACCARINI P, GRAZI GL, MANCINI AM: Overexpression of p53 protein and Ki67 proliferative index in hepatocellular carcinoma: an immuno-histochemical study on 109 Italian patients. Pathol Int 1994, 44:682-687.

6. NAKANISHI K, SAKAMOTO M, YAMASAKI S, TODO S, HIROHASHI S: Akt phosphorylation is a risk factor for early disease recurrence and poor prognosis in hepatocellular carcinoma. Cancer 2005, 103:307-312.

7. FARINATI F, CARDIN R, DERRICO A et al: Hepatocyte proliferative activity in chronic liver damage as assessed by the monoclonal antibody MIB1 Ki-67 in archival material: the role of etiology, disease activity, and iron and lipid peroxidation. Hepatology 23: 1468-1475, 1996.

8. SOYUER I., EKUNCU C., KAYA M., GEN Y.: Diagnostic Value of Ki-67LI in Hepatocellular Carcinoma Turk J Med Sci 33 (2003) 15-19. 